\title{
LA MIRADA DE LA ESCUELA A LA INTERCULTURALIDAD: EL CIMIENTO DE LA CIUDADANÍA ${ }^{1}$
}

\author{
Mํㅗㄴ Soledad Ortega Velasco ${ }^{2}$
}

\begin{abstract}
A total of 91 immigrants who have passed through the Spanish educational system tell us about their experience at school and how it has served them. We could say that it is important that our schools are open to new things and to the future because we are educating today the citizens of tomorrow. The teacher's role is paramount, so his/her training must nurture concepts such as cultural hybridization and the pedagogy of hybridization.
\end{abstract}

Keywords: intercultural perspective; intercultural education; citizenship; classroom relationships; teacher education; professional identity

Resumen: Un total de noventa y un inmigrantes que han pasado por el sistema educativo español nos cuentan cómo ha sido su experiencia y para qué les ha servido la escuela. En base a esto se puede decir que es muy importante que la mirada en la escuela sea cercana, abierta a lo nuevo y con proyección de futuro, ya que en ella se educan hoy a los ciudadanos del mañana. El papel que en esta labor asume el docente es fundamental, por lo que deben incorporarse en su formación conceptos como el de hibridación cultural o el de pedagogía de la hibridación.

Palabras clave: interculturalidad; educación intercultural; ciudadanía; la relación en el aula; formación del profesorado; identidad profesional

Bajo el enunciado "la mirada de la escuela a la interculturalidad" pueden esconderse muchas realidades, muchos conceptos y muchas prácticas y en base a éstas surgen las preguntas: ¿a qué nos referimos con "escuela"?, ¿cómo concretamos su "mirada"?, ¿cómo entendemos la "interculturalidad" y cómo la abordamos? Pues bien, en esta ocasión vamos a concretar esa mirada de la escuela en la dinámica académica y social que se establece en los centros educativos; y la interculturalidad la consideraremos desde el alumnado inmigrante que ha pasado por nuestras aulas en los últimos 30 años. Así, al hablar de mirada de la escuela aterrizaremos en la relación y en el trato cotidiano que se establece entre las personas que conviven en los centros educativos (docentes, otro personal del centro, alumnado y familias), analizando la importancia que esto puede tener de cara a la inserción futura en la sociedad.

Ortega Velasco, $M^{a}$ S. (2011). La mirada de la escuela a la interculturalidad: el cimiento de la ciudadanía. DEDiCA. REVISTA DE EDUCAÇÃO E HUMANIDADES, 1 (2011) Março, 521-532 
Y no lo vamos a hacer desde cualquier óptica: vamos a analizar esa mirada desde el punto de vista de este alumnado inmigrante, en concreto del de noventa y una personas (cuarenta y siete mujeres $-51,6 \%-$ y cuarenta y cuatro hombres $-48,4 \%-)$ con el siguiente perfil: han nacido fuera de nuestro país, en la actualidad están residiendo en la provincia de Málaga, son ya mayores de edad y han realizado al menos un curso en la etapa obligatoria del sistema educativo español ${ }^{3}$.

Al preguntarles sobre cómo recuerdan su experiencia escolar, nos encontramos con que 77 de ellos (84,6\%) la recuerdan agradable, siete de ellos $(7,7 \%)$ la recuerdan desagradable y otros tantos la recuerdan en parte agradable y en parte desagradable. Podría decirse que la mayoría de ellos, casi un $85 \%$, ha percibido la mirada de la escuela como agradable.

También hemos preguntado si se sentían valorados en la escuela española. 79 han contestado afirmativamente, (65 afirman que se han sentido valorados tanto por los profesores como por los compañeros, 10 solo por los profesores y 4 solo por los compañeros); 10 de ellos han contestado que no se han sentido valorados en la escuela española; 2 personas no han contestado a esta pregunta. Diríamos entonces que un $88,8 \%$ de las personas que han contestado a esta cuestión se han sentido valoradas en la escuela (por profesores, por compañeros o por ambos) frente a un $11,2 \%$ que no lo han hecho. Parece un resultado muy positivo $y$, en la misma línea que en la cuestión anterior, podría afirmarse que la mirada de la escuela ha sido recibida por este alumnado como cercana y atenta.

Si nos fijamos ahora en la actitud de las personas inmigrantes que llegan a nuestra escuela, nos encontramos con que solo 4 de estas personas no han tenido ningún interés por aprender mientras eran alumnos de la escuela española ${ }^{4}$. Muy al contrario, 84 de ellas sí han tenido ese interés, aunque en algunos casos (18) dependía del profesor, de la asignatura o de ambos.

Los resultados del análisis cuantitativo no nos permiten establecer relaciones entre las variables anteriores ya que los subgrupos de las personas que no se han sentido valoradas, que recuerdan la experiencia escolar como desagradable 0 que no muestran ningún interés por aprender son muy poco numerosos. Aún así podría sospecharse cierta relación entre sentirse valorado o valorada y el interés que se tiene por aprender, ya que resultaría estadísticamente significativo $(0,021)$ la relación entre sentirse valorado (por profesores y compañeros o por uno de los dos), y el 
interés por aprender: en concreto 59 de las 76 personas que se sentían valoradas (un $77,6 \%$ ) tenían interés por aprender. Por otra parte, la asignatura y el profesor dejan de ser un aliciente para aprender en aquellas personas que no se sentían valoradas $(0 \%)$.

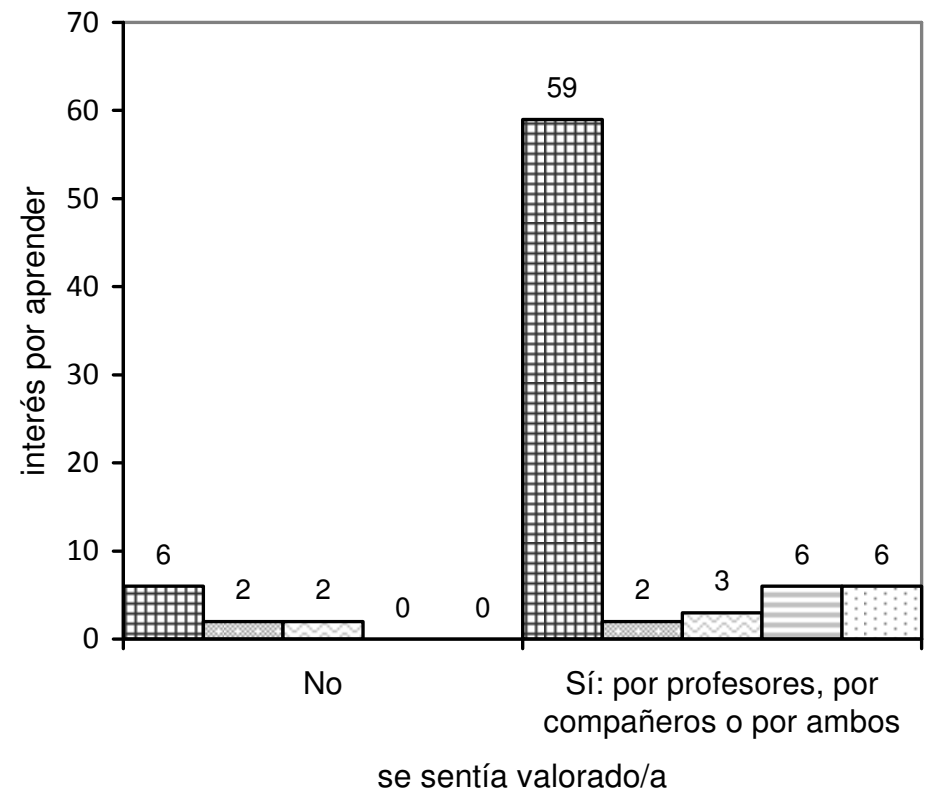

\begin{tabular}{|c|c|}
\hline 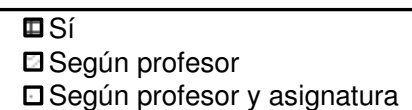 & $\begin{array}{l}\text { घNo } \\
\text { घSegún asignatura }\end{array}$ \\
\hline
\end{tabular}

Gráfico 1. Relación entre el sentimiento de valoración percibido en la escuela y el interés por aprender en la misma

Por todo esto, se defiende que esa mirada de la escuela debe ser delicada y transmitirse de una forma sensible, al menos, con el alumnado inmigrante.

Y esto no solo se defiende en esta intervención a corto plazo, sino que también se hace con vistas de futuro, ya que la escuela es de los primeros contextos sociales y españoles ante los que se enfrenta el alumnado extranjero o inmigrante que llega a nuestras aulas, y no es difícil pensar que, al salir de ella, su experiencia escolar habrá condicionado la forma en la que se dirigen y entienden a la sociedad de acogida. Para valorar la repercusión que esa mirada de la 
escuela, ese trato, esa acción educativa en todas sus dimensiones, ha tenido para ellos a medio y a largo plazo, en cómo se sitúan ante la sociedad española los inmigrantes que han pasado por sus aulas, les hemos preguntado sobre las siguientes cuestiones:

1. Sobre el nivel educativo adquirido: tan solo una persona de los 91 encuestados no tiene titulación, aunque sabe leer y escribir; el 13,2\% (12 personas) tiene estudios primarios, el $69,2 \%$ (63 personas) tiene estudios medios, el 16,5\% (15 personas) tiene estudios universitarios. Parece con ello que la escuela como institución que permite el acceso a estudios superiores ha supuesto una buena inversión de futuro para estas personas y para este país, ya que no podemos olvidar que todas estas personas residen actualmente en él.

2. Sobre si se sienten importantes para la sociedad española: encontramos que un $84,6 \%$ (77 personas) sí se sienten importantes para la sociedad española, frente a un $13,2 \%$ que no lo hace (12 personas), contando con dos personas que no saben qué contestar. Esta cuestión es muy importante, ya que nos da idea del papel que juegan en la sociedad española y de la conciencia que tienen de él ${ }^{5}$.

3. Sobre el sentirse ciudadano español: con esta pregunta complementamos la anterior, y observamos que un $76,7 \%$ de las personas encuestadas afirman sentirse ciudadanas españolas, independientemente de tener o no sus papeles arreglados o de haber adquirido la nacionalidad española (10 personas de 70 se encuentran en estos casos); el 23,3 \% (20 personas) afirma no sentirse ciudadanas españolas, a pesar de que cuatro de ellas tienen la nacionalidad española ${ }^{6}$. En este sentido vemos que un inmigrante puede ser perfectamente un ciudadano español y, lo que es más importante, sentirse como tal: la mayoría de las personas que llegaron a nuestro sistema educativo se sienten hoy ciudadanos, y trabajan y participan como cualquier persona autóctona. Esto nos hace pensar en que la etiqueta "inmigrante" va dejando paso a otras realidades sociales más complejas en las que una persona inmigrante no tiene por qué ser alguien ajeno a nuestra sociedad, sino que asume un papel mucho igual de activo que cualquier persona autóctona.

4. Sobre su proyecto de permanencia en este país: el $93 \%$ de las personas encuestadas (80 personas) tienen un proyecto de permanencia en nuestro país (72 de ellas volverían a su país de visita, y ocho se quedarían para siempre en España); un 7\% (6 
personas) volverían a su país definitivamente, y 5 personas no saben qué contestar. Estos datos sí llaman mucho la atención ya que la inmensa mayoría de las personas entrevistadas tienen un proyecto de permanencia en este país, independientemente del tiempo que lleven en él ${ }^{7}$, y nos hacen caer en la cuenta de que los inmigrantes que llegan a nuestras aulas hoy serán, en muchos casos, ciudadanos españoles del mañana, por lo que al plantearnos cómo queremos que sea la mirada que la escuela proyecta sobre ellos en la actualidad estamos diseñando el futuro de nuestra sociedad.

Es momento ahora de plantear un concepto muy importante que condiciona la dinámica del aula: el de "hibridación cultural"8. Resulta fácil hablar de una escuela para todos y que llegue a todos, que ha superado la etiqueta de "extranjero" o de "inmigrante" para concebir a su alumnado como personas que, independientemente de sus experiencias migratorias -y no por ello de espaldas a ellas-, han llegado a sus aulas para crecer como personas y ciudadanas españolas responsables. Esto solo se concibe enmarcando nuestras relaciones en un proceso de hibridación cultural por el que el alumnado, proceda de donde proceda, recibe de la cultura mayoritaria y aporta a la misma desde un plano de igualdad, generando así una identidad viva y compartida que crece y se alimenta de lo bueno que hay en cada persona.

Resulta fácil hablar de ello, pero no llevarlo a la práctica: es difícil que desde el primer responsable de la política educativa de nuestro tiempo hasta el último miembro del equipo educativo de la escuela rural más perdida de nuestras tierras piensen y conciban la escuela y la labor educativa en esta clave de humanidad.

$Y$ es que con este planteamiento se supera el tradicional concepto de interculturalidad centrado en el alumnado hijo de inmigrante, para entenderlo como un fenómeno social que afecta a toda persona que se relaciona en un contexto social determinado. Es decir, lo intercultural es una variable más inherente a cualquier acto educativo.

Por tanto, todo lo que pueda decirse sobre la educación, sobre la educación intercultural o sobre el sistema educativo en general es válido tanto para la persona autóctona como para la inmigrante, para la extranjera o para la que está en situación irregular; ya que, en una sociedad en la que la inmigración es parte consustancial de la misma, no cabe más distinción que la jurídico-administrativa en la condición de inmigrante o extranjero. Es decir, la inmigración configura tanto a la 
sociedad española actual, el fenómeno migratorio la está cambiando tanto, que nuestras escuelas deben concebir la interculturalidad en un plano de normalidad, como un fenómeno social que forma parte de nuestra cultura, de nuestra idiosincrasia y de la manera de estar abiertos al mundo en sociedades democráticas como la nuestra.

Es necesario, por tanto, no sólo tender puentes hacia la interculturalidad, sino superarlos y concebir el fenómeno intercultural en sí como parte inherente de nuestra propia cultura. Ser español no es solo conocer a españoles y convivir con españoles, es encandilar con tu forma de ser y de estar, al tiempo que te dejas encantar por todo lo bueno que de otras personas, compañeros y amigos pueda llegar, independientemente de su proceso migratorio. En otras palabras: es abrir al cambio lo superficial y, en caso necesario, también lo nuclear de la propia identidad. En esto consiste superar el tradicional concepto de interculturalidad y caminar hacia la hibridación cultural; y es necesario este cambio de mentalidad en cualquier agente educativo para seguir dando respuestas desde el sistema educativo a una sociedad intercultural en permanente cambio. En esto, la formación inicial y permanente del profesorado tiene mucho camino por andar.

Con este enfoque se está diciendo que no sean necesarias medidas específicas para atender al alumno inmigrante como pueden ser la relacionadas con el lenguaje, ni que el profesorado no tenga que tener una preparación específica que le ayude a abordar profesionalmente a esta realidad social; muy por el contrario, todo esto es fundamental, si bien es cierto que en la actualidad nuestro sistema educativo ya ha establecido estas medidas y maneras de hacer en su cotidianeidad. Es por esto, por este buen hacer de la escuela y de sus profesionales, por lo que nos atrevemos a dar este paso más en la concepción de la interculturalidad, animando a que lo hagan aquellos centros educativos y aquellos docentes que aún no abordan con naturalidad y eficacia este fenómeno.

Volvamos ahora a los datos presentados. Si buscamos alguna relación relevante entre estas cifras, que de por sí son muy alentadoras, y las variables anteriores relacionadas con la experiencia en la escuela, encontramos estadísticamente significativa la relación entre el sentirse valorado y el nivel educativo adquirido a lo largo del tiempo, con un p-valor de 0,05. 


\begin{tabular}{|l|l|l|l|}
\hline & $\begin{array}{l}\text { No se sentía } \\
\text { valorado/a }\end{array}$ & $\begin{array}{l}\text { Se sentía } \\
\text { valorado/a }\end{array}$ & Total \\
\hline Estudios universitarios & $28,6 \%$ & $71,4 \%$ & $100 \%$ \\
$(4$ personas $)$ & $(10$ personas $)$ & $(14$ personas $)$ \\
\hline Estudios medios & $6,5 \%$ & $93,5 \%$ & $100 \%$ \\
& $(4$ personas $)$ & $(58$ personas $)$ & $(62$ personas $)$ \\
\hline Nivel básico (leer y escribir & $11,2 \%$ & $84,6 \%$ & $100 \%$ \\
o estudios básicos) & $(2$ personas $)$ & $(11$ personas $)$ & $(13$ personas $)$ \\
\hline Total & $11,2 \%$ & $88,8 \%$ & $100 \%$ \\
& $(10$ personas) & $(79$ personas) & $(89$ personas $)$ \\
\hline
\end{tabular}

Tabla 1. Relación entre el sentimiento de valoración percibido en la escuela y el nivel educativo adquirido a lo largo del tiempo.

De ahí, y siguiendo la misma línea de las conclusiones anteriores, podríamos afirmar que es imprescindible que esa mirada de la escuela sobre el alumnado inmigrante deje su huella en estas personas, y las haga sentirse aceptadas y valoradas. Sin quitar importancia al grupo de compañeros de cara a que una persona se sienta más o menos valorada en el centro educativo, en esto cobra un papel fundamental el profesorado: la dinámica que se establece en el aula, su forma de ver, entender y de tratar "al otro", el reconocimiento del esfuerzo que hace el alumnado, y con esta actitud, el tipo de mensajes que les llegan por parte del docente son, entre otros factores, claves para provocar que el alumnado se sienta valorado en el aula y, con ello, que adquiera confianza para finalizar sus estudios $y$, en muchos casos, continuar con estudios avanzados. Para todo ello y considerando la intervención con personas procedentes de diferentes culturas, son destacables la concepción de la interculturalidad del docente, las ideas previas que tenga el profesor sobre su alumnado y sobre su procedencia cultural, y las expectativas que proyecte sobre éste. Lo que hay en el fondo de todo esto es una cuestión de identidad profesional, no sólo a nivel de primaria sino también de secundaria (muy importante ahora con la reforma de los nuevos planes de estudio). Es necesario reenfocar desde estos nuevos conceptos el papel del educador: qué pretende con sus alumnos, cómo entiende su actividad, qué papel juega ante la escuela y la sociedad, qué mirada aporta a la institución escolar y qué mirada proyecta sobre el alumnado, sobre sus familias y sobre cualquier otro profesional del centro. En definitiva, cada profesional se concibe a sí mismo y al concepto de interculturalidad de una forma y es necesario que sea consciente de que esa concepción tiene una gran repercusión en el trato sobre los demás; así, es necesario que su identidad profesional lo lleve a tratar con delicadeza y respeto a todo el alumnado, y más aún al 
que más lo necesita o al que más perdido pueda encontrarse, como es el caso de la mayoría del alumnado inmigrante al llegar a nuestras aulas. $Y$ es que su manera de entenderse a sí mismo influirá en la forma en la que trata a su alumnado y viceversa, en la manera en la que éste se dirige a él como docente y como maestro; marcará así la percepción que el alumnado tenga sobre sí mismo y sobre su importancia como alumno, como persona y, en el fondo, como ciudadano o ciudadana de nuestro país. Podríamos concluir con que es necesario este cambio de perspectiva docente: la educación intercultural es ya parte misma de la educación, y en cualquier caso, de lo que se trata es de formar a ciudadanos formados, seguros de sí mismos, responsables y activos ${ }^{10}$ que sean capaces de comprometerse con la mejora de una sociedad como la nuestra, y, ampliando la visión, con la mejora del mundo en general. Tanto desde la formación inicial como de la permanente hay que seguir trabajando por conseguir que esta mentalidad de dejar de ver al otro como diferente ahonde en la identidad del docente del siglo $\mathrm{XXI}$.

Muchos de nuestros docentes piensan así, y lo han estado haciendo al menos durante los últimos cuarenta años. De no ser así, no tendríamos en nuestras manos los datos de los que hemos estado hablando hasta ahora. Aún así, y en base a las entrevistas realizadas con estos docentes ${ }^{11}$, queda mucho por hacer.

Una cuestión importante que no puede quedarse atrás y que debe impregnar la mentalidad de cualquier docente y compañero de aula es la relativa a mantener una mirada abierta de cara a lo nuevo que puede llegar a nuestras aulas a través de este tipo de alumnado: la mirada de la escuela, concretada en la actuación de los profesionales que en ella trabaja y en la dinámica establecida entre compañeros y entre docentes y discentes, debe estar alerta para incorporar en el acervo cultural compartido y re-creado en el centro todos aquellos valores, actitudes y comportamientos que puedan servir para el enriquecimiento y crecimiento del grupo. Se incorpora así una nueva idea fundamental: los valores no son únicos, y si bien es cierto que no todo vale, es fundamental mantener una reflexión continua sobre los valores aceptados y mantenidos individual $\mathrm{y} / \mathrm{o}$ colectivamente, manteniendo $\mathrm{e}$ incorporando aquellas actitudes, valores y comportamientos que hacen al ser humano más feliz. El fenómeno intercultural nos lo recuerda una y otra vez. $Y$ es que en esto consiste, al final, ese 
enriquecimiento mutuo que sólo es posible desde la horizontalidad que aporta el concepto de hibridación cultural ${ }^{12}$.

Pongamos un ejemplo de lo que estamos hablando: una aportación que hacen muchos de los inmigrantes con los que nos hemos entrevistado es la del respeto: les sorprende mucho la forma de tratar al profesor que tienen los chicos y chicas que viven aquí, y la manera en la que los profesores tratan a su alumnado. En muchos casos ésta es la diferencia más significativa que encontraban entre la escuela de origen y la española. Por ejemplo, a una chica francesa de 37 años ${ }^{13}$ le sorprendía tremendamente que los profesores le dijeran en clase cuando era alumna "a ver, tú, sal a la pizarra". En la escuela francesa la relación entre los profesores y el alumnado se establecía con mucho respeto, según nos cuenta, pero no con un respeto impuesto que genera tensión, sino como una forma de tratarse natural y normal. Estaba acostumbrada a que se dirigieran con el término "señorita" y su apellido; eso era "lo normal", todos se trataban así y entre los niños también. $Y$ es que las fórmulas de cortesía en el trato son un aspecto nuclear de la cultura francesa, y nos da una idea del papel que el inmigrante puede asumir de forma activa en la sociedad, defendiendo con ello valores de primera necesidad que, en muchos casos tienden a quedar en segundo plano nuestra cultura.

Aún así, al llegar a España y ver que en la relación en el aula es diferente, es fácil que se produzca una adaptación aprovechando las "ventajas" que para un alumno puede tener esa situación. Por ejemplo, otro chico francés de 34 años nos comenta que él sacó mucho partido al hecho de que pudiera "salir del instituto a desayunar sin que nadie te controle". En palabras de Gimeno, "lo ajeno se suma a lo propio, lo enriquece, lo cuestiona, lo anula o le da otra perspectiva" (Gimeno, 2001: 89). También puede darse el caso en el que "ser respetuoso con los demás" no sea algo aceptado entre el grupo de iguales, por lo que la persona abandone este tipo de valores para poder sentirse integrado en su grupo de amigos. Es el caso de un chico de 26 años nacido en Hong Kong, de nacionalidad británica, y que lleva 19 años en España. Según nuestras entrevistas, estas modificaciones del comportamiento parecen producirse en rasgos periféricos de la identidad, ya que con el tiempo la forma de entender el respeto y las relaciones se mantienen. Por ejemplo, la chica francesa de la que hablábamos tiene una hija en edad escolar actualmente, y ella manifiesta que procura que su hija interiorice y ponga en práctica con sus 
profesores el comportamiento respetuoso hacia los demás, especialmente hacia los mayores, y le insiste en que sea cortés y amable con su profesores y profesoras aunque no tengan razón en lo que plantean.

Por lo tanto, valorando la situación actual de nuestra sociedad en torno a muchas de las relaciones de respeto que en ella se establecen, es esperanzador pensar en un cambio en la forma dominante de sociabilidad ${ }^{14}$ que impera en esta dimensión del respeto. $Y$ si nos paramos a analizar la estabilidad con la que se asienta el valor de respeto en una persona inmigrante que lo ha integrado como parte nuclear de su identidad, podemos imaginarnos el papel que los inmigrantes extranjeros pueden asumir si se comportan como ciudadanos activos y defienden con su actuar diario este valor cívico de primera necesidad en sociedades democráticas como la nuestra. En todo esto la escuela tiene un papel muy importante: el de saber ver y aprovechar lo bueno que cada alumno en general, y el inmigrante en particular, pueda aportar al grupo. En este sentido es fundamental que la mirada de la escuela a la interculturalidad, además de cercana sea abierta.

Como conclusión final, decir que dada la realidad de nuestra sociedad, podríamos estar de acuerdo en que nuestro sistema educativo en general y la escuela en particular no pueden permitirse ni una mirada distante, ni pasiva ni poco influyente en nuestros días: nuestra sociedad necesita de instituciones educativas que sean cercanas a las necesidades de su alumnado y de sus familias, que suplan la carencia de valores y de actitudes positivas en nuestros días y que incidan en el desarrollo de personas felices, competentes y capacitadas para el ejercicio de la ciudadanía.

Si consideramos el enfoque de la interculturalidad hasta aquí planteado, esto es, que en nuestra sociedad se desarrollara un proceso de hibridación cultural y que la educación se pensara en clave de humanidad en todos sus niveles y dimensiones, lo dicho para el sistema educativo y para la escuela no sólo es válido para el alumnado inmigrante o para el extranjero, sino también para el autóctono.

$Y$ es que, independientemente del proceso migratorio del alumnado, es una inversión de futuro que la mirada de la escuela sobre éste sea cercana, abierta a lo desconocido y bueno del otro, comprometida y eficaz en el desarrollo de ciudadanos agradecidos a la sociedad de procedencia o de acogida, según sea el caso. No se puede perder de vista esa proyección de futuro en la mirada de la escuela: estamos formando o educando y tratando con los 
ciudadanos del mañana, andamos forjando un futuro que nosotros aún no conocemos pero que sí proyectamos y deseamos. Desde esta perspectiva la escuela es un agente de desarrollo de esta hibridación cultural deseable, al tiempo que un instrumento generador de ciudadanía (Gimeno, 2001: 159), que forma el juicio y el comportamiento de los ciudadanos hacia la convivencia pacífica y democrática propia de nuestras sociedades contemporáneas.

\section{Bibliografía}

Bartolomé, M. (Coord.) (2002). Identidad y ciudadanía. Un reto a la educación intercultural. Madrid: Narcea.

Colectivo loé. Intervención Sociológica (2011). Consultado en 8 de marzo de 2011. Disponible en: http://www.colectivoioe.org/

Gimeno, J. (2001). Educar y convivir en la Cultura Global. Madrid: Morata.

Gimeno, J. (1982). Autoconcepto, sociabilidad y rendimiento escolar. Madrid: Instituto Nacional de Ciencias de la Educación.

Kymlicka, W. (1995). Ciudadanía multicultural. Barcelona: Paidós.

Naval, C. (2000). Educar ciudadanos. La polémica liberalcomunista en educación. Navarra: Eunsa.

Ortega, M. S. (2009). Una reflexión sobre el derecho a una educación para todos. En VV. AA., Actas del VI Congreso Internacional de Filosofía de la Educación, 261-270. Dykinson: Madrid.

Ortega, M. S.; Rascón, T. (2008). Los inmigrantes hablan: la escuela y la adquisición del concepto de ciudadanía. En VV. AA., Comunicaciones al XIV Congreso Nacional y III Iberoamericano de Pedagogía, 1311-1320. Madrid: Sociedad Española de Pedagogía.

Ortega, M. S. (2008). Reflexiones extranjeras, reflexiones ciudadanas. Addenda al XXVII Seminario Interuniversitario de Teoría de la Educación "Educación y ciudadanía". Consultado en 25 de enero de 2011. Disponible en: http://www.ucm.es/info/site/docu/27site/ad307.pdf

\footnotetext{
The importance of schools regarding interculturality: the foundation of citizenship

${ }^{2}$ Doctoranda.

Universidad de Málaga (España).

Email: sortega@uma.es

${ }^{3}$ En este perfil se incluyen tanto inmigrantes como extranjeros como personas en situación irregular, entendiendo el concepto de inmigración como la condición social de la población que ha llegado a España procedente de otros países. Para concretar su uso y evitar su abuso, decir que se incluirían como "extranjeras" a las personas que adoptaron la nacionalidad española tras emigrar, haciendo referencia a una cuestión de índole administrativa-jurídica. El inmigrante, desde este punto de vista, no tendría adquirida la nacionalidad española. Así, el concepto de inmigración aludiría a
} 
la condición social de la población que ha llegado a España procedente de otros países (Colectivo loé. Intervención Sociológica, 2011).

${ }^{4}$ Tres personas no han contestado esta pregunta.

5 Quedaría por estudiar qué otras variables están influyendo en estos aspectos estudiados para el medio y largo plazo, cuestión que no procede en este momento para esta intervención, pero que se está realizando en el actual proyecto de investigación del Grupo de Investigación GHUM 169 del Departamento de Teoría e Historia de la Educación.

${ }^{6}$ Para profundizar en el concepto de ciudadanía y en el sentimiento de pertenencia a la sociedad española puede consultarse la comunicación al XIV Congreso Nacional y III Iberoamericano de pedagogía (Ortega, 2008).

${ }^{7}$ El número de años que llevan viviendo en España las personas entrevistadas varía entre 1 y 37, presentando mayor número de casos entre 3 y 9 años. La medida de estancia en España sería de 12,5 años, con una desviación típica de 9,0 asimetría hacia la izquierda (skewness 0,86 ) y cierto apuntamiento en torno a los 10 años (Kurtosis -0.54).

${ }^{8}$ La hibridación cultural consiste en la existencia de una interacción desordenada entre diferentes culturas que han dejado de situarse desde distintos puestos de poder para situarse en un plano de igualdad. En base a este concepto se habla de una pedagogía de la hibridación, que persiguen mantener un equilibrio entre lo propio y lo ajeno (Gimeno, 2001:99).

${ }^{9}$ Este "otro" se entiende en sentido general: tanto al otro como persona (cualquier tipo de alumnado) como al otro como persona procedente de otra cultura. En ambos casos "el otro" tiene una forma de entender y de tratar al mundo que le rodea de manera diferente al propio docente. Somos diferentes, interpretamos diferente, pensamos diferente... aún viviendo en un mismo contexto.

${ }_{10}$ Para profundizar en el concepto de "ciudadanía activa" puede consultarse el libro de Margarita Bartolomé (Bartolomé, 2002: 89).

${ }^{11}$ Están siendo estudiadas en la actualidad por el grupo de investigación GHum 169 del Departamento de Teoría e Historia de la Educación.

${ }^{12}$ Cuestiones como éstas serían las que se abordarían desde la Pedagogía de la Hibridación que deriva de este planteamiento de la hibridación cultural.

${ }^{13}$ Chica nacida en Francia, con nacionalidad española y francesa, que lleva 26 años en España.

14 J. Gimeno (Gimeno, 1982) distingue diversas modalidades de sociabilidad en función de la densidad de las relaciones que establece la persona, pudiendo ser afectivas, políticas y jurídicas, de solidaridad, de cooperación, culturales, o de los extraños (no relaciones). 